\title{
Localization in the Ground State of a Disordered Array of Quantum Rotators
}

\author{
Abel Klein ${ }^{1}$ and J. Fernando Perez ${ }^{2}$ \\ Department of Mathematics, University of California at Irvine, Irvine, CA 92717, USA
}

Received July 25, 1991; in revised form December 19, 1991

Abstract. We consider the zero-temperature behavior of a disordered array of quantum rotators given by the finite-volume Hamiltonian:

$$
H_{\Lambda}=-\sum_{x \in \Lambda} \frac{h(x)}{2} \frac{\partial^{2}}{\partial \varphi(x)^{2}}-J \sum_{\langle x, y\rangle \in \Lambda} \cos (\varphi(x)-\varphi(y)),
$$

where $x, y \in \mathbf{Z}^{d},\langle$,$\rangle denotes nearest neighbors in \mathbf{Z}^{d} ; J>0$ and $\mathbf{h}=\{h(x)>0$, $\left.x \in \mathbf{Z}^{d}\right\}$ are independent identically distributed random variables with common distribution $d \mu(h)$, satisfying $\int h^{-\delta} d \mu(h)<\infty$ for some $\delta>0$. We prove that for any $m>0$ it is possible to choose $J(m)$ sufficiently small such that, if $0<J<J(m)$, for almost every choice of $\mathbf{h}$ and every $x \in \mathbf{Z}^{d}$ the ground state correlation function satisfies

$$
\langle\cos (\varphi(x)-\varphi(y))\rangle \leqq C_{x, \mathbf{h}, J} e^{-m|x-y|}
$$

for all $y \in \mathbf{Z}^{d}$ with $C_{x, \mathbf{h}, J}<\infty$.

\section{Introduction}

Ferromagnetically coupled quantum rotators have been used in the physics literature to describe the effect of quantum fluctuations in granular superconductors [1]. In this paper we discuss the typical properties of a disordered array of such rotators with random moments of inertia. Apart from its intrinsic physical interest the study of this model is a natural step in the program initiated in [2] and [3] of understanding the effect of randomness in quantum spin systems. In [2], Klein and Perez studied the ground state of the one-dimensional quantum $x-y$ model in the presence of a random transverse field: exponential decay of the correlation

\footnotetext{
1 Partially Supported by NSF under grants DMS 8905627 and INT 8703059

2 Permanent Address: Instituto de Fisica, Universidade de Sao Paulo, P.O. Box 20516, 01498 Sao Paulo, Brazil. Partially Supported by CNPq under grant 303795-77FA
} 
functions for any amount of disorder was shown to be a consequence of exponential localization for one-dimensional random Schrödinger operators. In [3] Campanino, Klein and Perez considered the $d$-dimensional Ising model in the presence of a random transverse field. A path space representation was used to map the original quantum model into a limit of a classical ferromagnetic Ising model in $(d+1)$-dimensions with $d$-dimensional disorder. This allowed the control of the Griffiths' type of singularities through the use of correlation inequalities and a multiscale analysis of the type used in the theory of localization for random Schrödinger operators $[4,5]$. Their results were: exponential decay of correlation functions for high-disorder and any $d \geqq 1$ and long-range order in the low disorder regime for $d \geqq 2$. For $d=1$ long range order at low disorder was established by Aizenman, Klein and Newman [13].

The ideas and techniques of this paper share much in common with those involved in [3], namely an approximate path space is used mapping the system into a limit of $(d+1)$-dimensional ferromagnetic classical rotators with $d$-dimensional disorder, allowing the use of correlation inequalities. The novel feature is the presence of a continuous symmetry which allows, through the use of McBryanSpencer bounds, an easier control of the Griffiths' singularities. Our multi-scale analysis follows the strategy of von Dreifus and Spencer [4], with the role of the resolvent identity replaced by the Simon-Lieb-Rivasseau inequality [10] and the role of Wegner's estimate replaced by McBryan-Spencer bounds.

\section{The Model and Results}

In a finite volume $\Lambda \subset \mathbf{Z}^{d}$ the Hamiltonian is given by

$$
H_{\Lambda}=-\sum_{x \in \Lambda} \frac{h(x)}{2} \frac{\partial^{2}}{\partial \varphi(x)^{2}}-J \sum_{\langle x, y\rangle \in \Lambda} \cos (\varphi(x)-\varphi(y))
$$

acting on the Hilbert space $\mathscr{H}_{\Lambda}=\otimes_{x \in \Lambda} \mathscr{H}_{x}, \mathscr{H}_{x}=L^{2}[-\pi,+\pi], x \in \mathbf{Z}^{d}$. The operator $-\frac{\partial^{2}}{\partial \varphi(x)^{2}}$ is taken with periodic boundary condition so that its spectrum is $\left\{n_{x}^{2}, n_{x} \in \mathbf{Z}\right\}$. The second sum in (2.1) is taken over all pairs of nearest neighbor sites $\langle x, y\rangle$ in $\Lambda$. The coupling between the rotators is ferromagnetic, i.e., $J>0$. The inverse of the moments of inertia of the rotators, $\mathbf{h}=\left\{h(x)>0, x \in \mathbf{Z}^{d}\right\}$, are taken to be independent identically distributed random variables with common distribution $d \mu(h)$. We shall allow $h(x)$ to take arbitrarily small, but positive values, with the condition that for some $\delta>0$,

$$
\frac{1}{\bar{h}^{\delta}} \equiv \int \frac{1}{h^{\delta}} d \mu(h)<\infty .
$$

We shall denote by $\mathbf{P}$ and $\mathbf{E}$ the underlying expectation and probability measure induced by $d \mu$. The parameter $\bar{h}$ defined by (2.2) measures the amount of disorder in the system.

The operator $H_{\Lambda}$ has a unique ground state $\Omega_{\Lambda}$, with a normalized wave function $\Omega_{\Lambda}(\varphi)>0$ for all $\varphi=\{\varphi(x), x \in \Lambda\}, \varphi(x) \in[-\pi,+\pi]$. This follows from the fact that $H_{A}$ has discrete spectrum and generates a positivity improving semigroup (Sect. 3). 
Moreover the correlation functions:

$$
\langle\cos (\varphi(x)-\varphi(y))\rangle_{\Lambda} \equiv\left(\Omega_{\Lambda}, \cos (\varphi(x)-\varphi(y)) \Omega_{\Lambda}\right)
$$

are monotonically increasing in $\Lambda$ and $J>0$ and monotonically decreasing in each $h(x), x \in \mathbf{Z}^{d}$. This follows from correlation inequalities derived in the path space representation (see Sect. 3).

The Hamiltonian (2.1) in the deterministic homogeneous situation $h(x) \equiv h$, for all $x \in \mathbf{Z}^{d}$, has been used to describe quantum fluctuations in superconducting arrays [1]. In its ground state for $d \geqq 2$, it exhibits a phase transition with long range order for $\alpha=\frac{J}{h}>\alpha_{c}(d)$. This follows from the path space representation developed in Sect. 3 and standard techniques [6]. In $d=1$, the system has a Kosterlitz-Thouless phase transition (see [14] for a similar gauge field model) with polynomial decay of correlation functions for $\alpha>\alpha_{c}(1)$. Mean field bounds (see Sect. 5) also guarantee the existence of $\bar{\alpha}_{c}(d), \alpha_{c}(d) \geqq \bar{\alpha}_{c}(d) \geqq \frac{1}{4 d}$, such that if $\alpha<\bar{\alpha}_{c}(d)$ the correlation functions decay exponentially.

In order to state our results we introduce the imaginary time correlation function

$$
G_{\Lambda}((x, t),(y, s))=\frac{\left(\Omega_{\Lambda}, e^{i \varphi(x)} e^{-|t-s| H_{\Lambda}} e^{-i \varphi(y)} \Omega_{\Lambda}\right)}{\left(\Omega_{\Lambda}, e^{-|t-s| H_{\Lambda}} \Omega_{\Lambda}\right)} .
$$

Monotonicity in $\Lambda$ (obtained from the path-space representation) ensures the existence of

$$
G((x, t),(y, s))=\lim _{\Lambda \rightarrow \mathbb{Z}^{d}} G_{\Lambda}((x, t),(y, s)),
$$

in terms of which we state our main result.

It is important to notice that if for some $\varepsilon>0$ we have $\alpha(x)=\frac{J}{h(x)}$ $\geqq(1+\varepsilon) \alpha_{c}(d)$ for all $x \in \mathbf{Z}^{d}$ with probability one, then the system will have long range order $(d \geqq 2)$ or polynomial decay of correlations $(d=1)$, with probability one. This is a consequence of monotonicity of $G((x, t),(y, s))$ in each $\alpha(x), x \in \mathbf{Z}^{d}$, and the corresponding result in the homogeneous deterministic case. Conversely if for some $\varepsilon>0, \alpha(x)<(1-\varepsilon) \bar{\alpha}_{c}(d)$, for all $x \in \mathbf{Z}^{d}$ with probability one, correlation functions will decay exponentially with probability one.

From the above it follows that non-trivial behavior is expected only when both $\alpha(x)>\alpha_{c}(d)$ and $\alpha(x)<\bar{\alpha}_{c}(d)$ may occur with non-zero probability.

Theorem 2.1. Let $d=1,2, \ldots, q>(1+3 / \delta) d+1$. Then for any $m>0$ there exists $J(m)$ such that, for any $0<J<J(m)$ and almost every choice of $\mathbf{h}$ and every $x \in \mathbf{Z}^{d}$, we have

$$
G((x, t),(y, s)) \leqq C_{x, \mathbf{h}, J} e^{-m i\left(x-y,|t-s|^{1 / 9}\right) \|_{x}},
$$

where $\|(x, u)\|_{\infty}=\max (|x|,|u|)$ and $C_{x, \mathbf{h}, J}<\infty$.

It is important to notice the less than exponential decay in the time direction compared with the exponential decay in the space direction. This is a consequence 
of the Griffiths' singularities, i.e., the fact that with probability one there exists arbitrarily large regions $\Lambda$ such that $\alpha(x)=\frac{J}{h(x)}>\alpha_{c}(d)$ for all $x \in \Lambda$. For these regions the energy gap between the ground state $\Omega_{\Lambda}$ and the first excited state gets arbitrarily small.

Remark. Our methods can actually prove a stronger result. We can admit random couplings $\left\{J_{\langle x, y\rangle} ;\langle x, y\rangle \in \mathbf{Z}^{d}\right\}$ and relax our hypothesis on the probability distribution of $h(x)$. More precisely, let

$$
H_{\Lambda}=-\sum_{x \in \Lambda} \frac{h(x)}{2} \frac{\partial^{2}}{\partial \varphi(x)^{2}}-J \sum_{\langle x, y\rangle \in \Lambda} J_{\langle x, y\rangle} \cos (\varphi(x)-\varphi(y)),
$$

where $J>0, \mathbf{h}=\left\{h(x)>0, x \in \mathbf{Z}^{d}\right\}$ and $\mathbf{J}=\left\{J_{\langle x, y\rangle}>0,\langle x, y\rangle \in \mathbf{Z}^{d}\right\}$ are independent sets of independent identically distributed random variables with

$$
\mathbf{E}\left([\log (1+1 / h(x))]^{\delta}\right)<\infty \text { and } \mathbf{E}\left(\left[\log \left(1+J_{\langle x, y\rangle}\right)\right]^{\delta}\right)<\infty,
$$

where $\delta>2 d$. In this case Theorem 2.1 still holds, with the conclusion being true of almost every choice of $\mathbf{h}$ and $\mathbf{J}$, and (2.5) replaced by

$$
G((x, t),(y, s)) \leqq C_{x, \mathbf{h}, \mathbf{J}} e^{-m \|\left(x-y,[\log (1+|t-s|)]^{q} \|\right.}
$$

for some $q>1$.

The modifications required in the proof are similar to the arguments in Klein [17].

\section{The Approximation by Classical Rotators}

Let us denote by $h_{0}$ the self-adjoint operator $-\frac{1}{2} \frac{d^{2}}{d \varphi^{2}}$ in $L^{2}[-\pi,+\pi]$ with periodic boundary condition. Our starting point is the formula

$$
K_{t}\left(\varphi, \varphi^{\prime}\right) \equiv e^{-t h_{0}}\left(\varphi, \varphi^{\prime}\right)=\frac{1}{\sqrt{2 \pi t}} \sum_{m \in \mathbf{Z}} e^{\frac{-\left(\varphi-\varphi^{\prime}+2 \pi m\right)^{2}}{2 t}}
$$

for the kernel of $e^{-t h_{0}}, t>0$.

Using (3.1) and the Trotter product formula we obtain the representation

$$
\left(\Omega_{\Lambda}, F\left(\varphi\left(x_{1}\right), \ldots, \varphi\left(x_{n}\right)\right) \Omega_{\Lambda}\right)=\lim _{n \rightarrow \infty}\left\langle F\left(\varphi\left(x_{1}, 0\right), \varphi\left(x_{2}, 0\right), \ldots, \varphi\left(x_{n}, 0\right)\right)\right\rangle_{A}^{(n)},
$$

where $\langle\cdot\rangle_{A}^{(n)}$ denotes the expectation for the classical plane rotator in $\Lambda \times \frac{\mathbf{Z}}{n} \subset \mathbf{Z}^{d} \times \frac{\mathbf{Z}}{n}$ (i.e., with lattice spacing $\frac{1}{n}$ in the "time" direction) with the so-called Villain approximation taken in the "time" direction i.e., the Gibbs weight of a configuration $\varphi=\left\{\varphi(x, t), x \in \Lambda, t \in \frac{\mathbf{Z}}{n} \cap\left[-\frac{\beta}{2}, \frac{\beta}{2}\right]\right\}$ given by

$$
e^{-H_{\lambda, \beta}^{(n)}(\varphi)} \equiv e^{\sum_{\langle x, y\rangle, t} \frac{J}{n} \cos (\varphi(x, t)-\varphi(y, t)} \sum_{\mathbf{m}} e^{-\frac{n}{2} \sum_{x, t} \frac{1}{h(x)}(\varphi(x, t)-\varphi(x, t+1 / n)+2 \pi m(x, t))^{2}},
$$


where $\mathbf{m}=\left\{m(x, t) ; x \in \Lambda, t \in\left[-\frac{\beta}{2},+\frac{\beta}{2}\right] \cap \frac{\mathbf{Z}}{n}\right\}$ and the summations are over $t \in\left[-\frac{\beta}{2},+\frac{\beta}{2}\right] ; x, y \in \Lambda$.

This approximation enables us to use Ginibre's correlation inequalities [9] taking into account the ferromagnetic nature of the model. This is made possible by the use of the remark [8] that

$$
\begin{aligned}
& F_{\beta}\left(\varphi-\varphi^{\prime}\right) \equiv \frac{\sum_{k \in \mathbf{Z}} \exp \left[-\frac{\beta}{2}\left(\varphi-\varphi^{\prime}+2 \pi k\right)^{2}\right]}{\sum_{k \in \mathbf{Z}} \exp -\frac{\beta}{2}(2 \pi k)^{2}} \\
& =\lim _{n \rightarrow \infty} c(n) \int_{[-\pi,+\pi]} d \theta_{1} \ldots \int_{[-\pi,+\pi]} d \theta_{n} \exp \left\{n \beta\left[\cos \left(\varphi-\theta_{1}\right)+\cdots+\cos \left(\theta_{n}-\varphi^{\prime}\right)\right]\right\}
\end{aligned}
$$

with suitably chosen $c(n)>0$. Formula (2.4) allows the substitution of the Villain couplings by standard rotator (cosine) couplings for which Ginibre's inequalities apply.

The derivation of (3.2) starts with the fact that the operator

$$
H_{\Lambda}^{(0)}=-\sum_{x \in A} \frac{h(x)}{2} \frac{\partial^{2}}{\partial \varphi(x)^{2}}
$$

has a unique ground state, given by the function

$$
\Omega_{\Lambda}^{(0)}(\varphi)=\frac{1}{(2 \pi)^{|\Lambda| / 2}}
$$

its spectrum being $\left\{\sum_{x \in A} \frac{h(x)}{2} l_{x}^{2}, l_{x} \in \mathbf{Z}\right\}$. Moreover the operator $H_{A}$ generates a positivity improving semigroup (this is true for $H_{A}^{(0)}$ from formula (2.1) and remains true for $H_{A}$ since $V_{A}(\varphi)=-J \sum\langle x y\rangle \in \Lambda \cos (\varphi(x)-\varphi(y))$ is a multiplication operator). Moreover, the spectrum of $H_{A}$ is discrete, since $H_{A}^{(0)}$ has compact resolvent and $V_{A}$ is bounded (e.g., [11, p. 113]). It follows from the PerronFrobenius theory [11] that $H_{\Lambda}$ has a unique ground state and $\Omega_{\Lambda}(\varphi)$ is a positive function. In particular

$$
\left(\Omega_{\Lambda}(\varphi), \Omega_{\Lambda}^{(0)}(\varphi)\right)>0 .
$$

It then follows for any bounded operator $A$ in $\mathscr{H}_{A}$ :

$$
\langle A\rangle_{\Lambda}=\left(\Omega_{\Lambda}, A \Omega_{\Lambda}\right)=\lim _{\beta \rightarrow \infty} \frac{\left(\Omega_{\Lambda}^{(0)}, e^{-\frac{\beta}{2} H_{\Lambda}} A e^{\frac{-\beta}{2} H_{\Lambda}} \Omega_{\Lambda}^{(0)}\right)}{\left(\Omega_{\Lambda}^{(0)}, e^{-\beta H_{\Lambda}} \Omega_{\Lambda}^{(0)}\right)} .
$$

Using Trotter's product formula we obtain [6]:

$$
\left\langle F\left(\varphi_{A}\right)\right\rangle_{\Lambda} \equiv\left(\Omega_{\Lambda}, F\left(\varphi_{A}\right) \Omega_{\Lambda}\right)=\lim _{\beta \rightarrow \infty} \lim _{n \rightarrow \infty}\left\langle F\left(\varphi_{A, 0}\right)\right\rangle_{\Lambda, \beta}^{(n)},
$$


where $\varphi_{A}=\{\varphi(x), x \in A\}, A \subset \Lambda ; \varphi_{A, t}=\left\{\varphi(x, t), x \in A, t \in \frac{\mathbf{Z}}{n}\right\}$. Here $\langle\cdot\rangle_{\Lambda, \beta}^{(n)}$ denotes the expectation in the classical rotator given by (3.3) and restricted to the region $\Lambda \times\left(\left[-\frac{\beta}{2}, \frac{\beta}{2}\right] \cap \frac{\mathbf{Z}}{n}\right)$ with free boundary conditions. Using Ginibre's inequalities (and the free boundary conditions) we get the monotonicity in the volume:

$$
\left\langle F\left(\varphi_{A}\right)\right\rangle_{\Lambda, \beta}^{(n)} \leqq\left\langle F\left(\varphi_{A}\right)\right\rangle_{A^{\prime}, \beta^{\prime}}^{(n)} \quad \text { if } \Lambda \subset \Lambda^{\prime}, \beta \leqq \beta^{\prime} .
$$

We may thus interchange the limits in (3.9):

$$
\left\langle F\left(\varphi_{A}\right)\right\rangle_{\Lambda} \equiv \lim _{n \rightarrow \infty}\left\langle F\left(\varphi_{A, 0}\right)\right\rangle_{\Lambda}^{(n)}
$$

In particular we can take the thermodynamical limit

$$
\left\langle F\left(\varphi_{A}\right)\right\rangle \equiv \lim _{\Lambda \rightarrow \mathbf{Z}^{d}}\left\langle F\left(\varphi_{A}\right)\right\rangle_{\Lambda}=\lim _{n \rightarrow \infty}\left(F\left(\varphi_{A}\right)\right\rangle^{(n)} \quad \text { for any } A \subset \mathbf{Z}^{d} .
$$

Correlation functions involving time can also be obtained. For instance

$$
\lim _{\Lambda \rightarrow \mathbf{Z}^{d}}\left(\Omega_{\Lambda}, F\left(\varphi_{A}\right) e^{-|t-s| H_{A}} G\left(\varphi_{B}\right) \Omega_{\Lambda}\right)=\lim _{n \rightarrow \infty}\left\langle F\left(\varphi_{A, t}\right) G\left(\varphi_{B, s}\right)\right\rangle^{(n)} .
$$

\section{An Estimate of the Energy Gap}

The existence of a continuous symmetry plays an important role in the estimate of the energy gap $E_{\Lambda}^{(1)}$ between the ground state $\Omega_{\Lambda}$ and the excited states in the invariant subspace generated by $\left\{e^{-i \varphi(x)} \Omega_{\Lambda}, x \in \Lambda\right\}$.

Such an estimate would give us a priori bounds on the decay of finite volume correlation functions, since it follows immediately from (2.4) that

$$
G_{\Lambda}((x, t),(y, s)) \leqq e^{-E_{A}^{(1)}|t-s|} .
$$

Let us introduce the total angular momentum operator

$$
L_{\Lambda}=\sum_{x \in \Lambda} \frac{1}{i} \frac{\partial}{\partial \varphi(x)} .
$$

The Hamiltonian can then be decomposed (e.g., [16, p. 77]) in the form of

$$
H_{\Lambda}=I_{\Lambda}^{-1} L_{\Lambda}^{2}+H_{\Lambda}^{r}
$$

where the first term is the "center of mass" Hamiltonian and the "relative" Hamiltonian $H_{\Lambda}^{r}$ involves only the relative coordinates $\{\varphi(x)-\varphi(y) ; x, y \in \Lambda\}$, and

$$
I_{\Lambda}=\sum_{x \in A} \frac{1}{h(x)}
$$

If we did not have periodic boundary conditions on $H_{A}^{(0)}$, our Hilbert space would be written as a tensor product with the two terms in (4.2) acting on different factors. It would then follow that $L_{\Lambda} \Omega_{\Lambda}=0$ and

$$
L_{\Lambda} e^{-i \varphi(x)} \Omega_{\Lambda}=e^{-i \varphi(x)}\left(L_{\Lambda}-1\right) \varphi_{\Lambda}=-e^{-i \varphi(x)} \Omega_{\Lambda}
$$


Thus

$$
L_{\Lambda}^{2} e^{-\imath \varphi(x)} \Omega_{\Lambda}=e^{-\imath \varphi(x)} \Omega_{\Lambda} .
$$

Therefore we would have

$$
E_{\Lambda}^{(1)} \geqq I_{\Lambda}^{-1} \geqq \frac{h_{\mathrm{min}}^{(\Lambda)}}{|\Lambda|},
$$

where $h_{\min }^{(A)}=\min _{x \in \Lambda} h(x)$. It would then follow from (4.1) that

$$
G_{\Lambda}((x, t),(y, s)) \leqq e^{-I_{A}^{-1}|t-s|} \leqq e^{\frac{-h_{\min }^{(1)}|t-s|}{|\Lambda|}} .
$$

The above argument is not correct since the periodic boundary conditions do not allow us to write our Hilbert space as a tensor product where each term in (4.2) acts on a different factor. But if all the $h(x)$ are rational numbers, this can be done in a bigger space where we can prove (4.3). The result then follows for arbitrary $h(x)$ by a perturbation argument.

This estimate should be compared with the estimate

$$
E_{\Lambda}^{(1)} \approx \prod_{x \in \Lambda}\left(\frac{h(x)}{J} \wedge 1\right) \geqq\left(\frac{h_{\min }^{(\Lambda)}}{J} \wedge 1\right)^{|\Lambda|}
$$

obtained for the Ising model in the presence of a random transverse field [3].

We actually need more than (4.4), we need a uniform bound on the correlation functions of the classical rotators given by (3.3). This is given by

Lemma 4.1. Let

Then

$$
G_{A, \beta}^{(n)}((x, t),(y, s))=\left\langle e^{i[\varphi(x, t)-\varphi(y, s)]}\right\rangle_{\Lambda, \beta}^{(n)} .
$$

$$
G_{\Lambda, \beta}^{(n)}((x, t),(y, s)) \leqq e^{-I_{A}^{-1}|t-s|} \leqq e^{-\frac{h_{\min }^{(A)}|t-s|}{|\Lambda|}}
$$

for all $\beta$ and $n$.

Proof. We use a technique of McBryan and Spencer [7].

Let $\alpha(t)$ be a $C^{(2)}$-function on $\mathbf{R}$. We perform the imaginary shift

$$
\varphi(x, t) \rightarrow \varphi(x, t)+i \alpha(t)
$$

on the integration variables appearing in the numerator of the expression for the correlation functions to obtain:

$$
\begin{aligned}
\left\langle e^{i[\varphi(x, 0)-\varphi(y, t)]}\right\rangle_{\Lambda, \beta}^{(n)} & \leqq e^{-[\gamma(0)-\gamma(t)]+n \sum_{x \in A} \sum_{s \in[-\beta, 2, \beta, 2] \cap} \frac{z}{n} \frac{(\alpha(s)-\alpha(s+1 / n))^{2}}{2 h(x)}} \\
& \leqq e^{-[\alpha(0)-\gamma(t)]+n\left(\sum_{x \in A n} 1 / h(x)\right) \sum_{s \in \frac{Z}{n}} \frac{1(\alpha(s)-\alpha(s+1 / n))^{2}}{2(1 / n)^{2}}} .
\end{aligned}
$$

We then choose

$$
\alpha=\frac{1}{\sum_{x \in A} \frac{1}{h(x)}}\left(-\frac{\partial^{2}}{\partial t^{2}}\right)_{n}^{-1}\left[\delta_{0}-\delta_{t}\right]
$$


where $\left(-\frac{\partial^{2}}{\partial t^{2}}\right)_{n}^{-1}$ is the inverse of the second difference operator (lattice Laplacian) in the lattice $\frac{\mathbf{Z}}{n}$, i.e.,

$$
\left[\left(-\frac{\partial^{2}}{\partial t^{2}}\right)_{n} f\right](t)=n^{2}\left[2 f(t)-f\left(t+\frac{1}{n}\right)-f\left(t-\frac{1}{n}\right)\right]
$$

given by

$$
\left(-\frac{\partial^{2}}{\partial t^{2}}\right)_{n}^{-1}(r, s)=\frac{1}{2}|r-s| \text { for } r, s \in \frac{\mathbf{Z}}{n}
$$

It follows that

$$
\left\langle e^{l[\varphi(x, t)-\varphi(y, s)]}\right]_{\Lambda, \beta}^{(n)} \leqq e^{-\frac{|t-s|}{\sum_{x \in A} \frac{1}{h(x)}}}
$$

proving the lemma.

\section{Mean Field Bounds}

An important feature of the classical path space model are the Simon-LiebRivasseau [10] inequalities. Let us state them in a form that is suitable for our purposes.

Let

$$
\Omega=\Lambda \times\left(\left[-\frac{\beta}{2}, \frac{\beta}{2}\right] \cap \frac{\mathbf{Z}}{n}\right) \subset \mathbf{Z}^{d} \times \frac{\mathbf{Z}}{n}, \quad W \subset \Omega .
$$

We shall denote by $\partial_{V} W$ the "vertical" interior boundary of $W$ i.e.,

$$
\partial_{V} W=\left\{(z, u) \in W: \exists\left(z^{\prime}, u\right) \notin W,\left|z-z^{\prime}\right|=1\right\},
$$

and by $\partial_{H} W$, the horizontal boundary of $W$, i.e.,

$$
\partial_{H} W=\left\{(z, u) \in W:\left(z, u+\frac{1}{n}\right) \text { or }\left(z, u-\frac{1}{n}\right) \notin W\right\} .
$$

With the notation $X=(x, t), Y=(y, s), Z=(z, u) \in \mathbf{Z}^{d} \times \frac{\mathbf{Z}}{n}$ we have:

$$
G_{\Omega}^{(n)}(X, Y) \leqq \sum_{Z \in \hat{\partial}_{H} W} G_{W}^{(n)}(X, Z) G_{\Omega}^{(n)}(Z, Y)+\frac{J}{n} \sum_{\substack{Z \in \partial_{V} W \\\left\langle Z, Z^{\prime}\right\rangle}} G_{W}^{(n)}(X, Z) G^{(n)}\left(Z^{\prime}, Y\right),
$$

where the second summation is taken over all $\left\langle Z, Z^{\prime}\right\rangle=\left\langle(z, u),\left(z^{\prime}, u\right)\right\rangle$ horizontal nearest neighbor pairs with $Z^{\prime} \notin W$.

Remark. In its original form Simon-Lieb-Rivasseau's inequality reads simply

$$
G_{\Omega}^{(n)}(X, Y) \leqq \sum_{Z \in \partial W} G_{W}^{(n)}(X, Z) G_{\Omega}^{(n)}(Z, Y)
$$


In order to shape it like in (5.1) one needs to apply Local Ward Identities $[6,16]$ to the bonds $\left\langle Z, Z^{\prime}\right\rangle$ crossing $\partial_{V} W$. We need them in the present form in order to control the limit $n \rightarrow \infty$.

Inequalities (5.1) will serve here a double purpose: to produce mean field bounds on $\bar{\alpha}_{c}(d)$ and give decay in the multiscale expansion.

Mean-field bounds are obtained by taking $W$ to be the thin set

$$
W_{l}(x, t)=\left\{(x, s) ; s \in I_{l}^{(n)}(t) \equiv\left[t-\frac{l}{2}, t+\frac{l}{2}\right] \cap \frac{\mathbf{Z}}{n}\right\} .
$$

In this case (5.1) reads

$$
\begin{aligned}
G_{\Omega}^{(n)}((x, t),(y, s)) \leqq & \sum_{\left|x-x^{\prime}\right|=1} \sum_{u \in I_{l}^{(n)}(t)} \frac{J}{n} G_{W_{l}(x, t)}^{(n)}((x, t),(x, u)) G_{\Omega}^{(n)}\left(\left(x^{\prime}, u\right),(y, s)\right) \\
& +G_{W_{l}(x, t)}^{(n)}\left((x, t),\left(x, t+\frac{l}{2}\right)\right) G_{\Omega}^{(n)}\left(\left(x, t+\frac{l}{2}\right),(y, s)\right) \\
& +G_{W_{l}(x, t)}^{(n)}\left((x, t),\left(x, t-\frac{l}{2}\right)\right) G_{\Omega}^{(n)}\left(\left(x, t-\frac{l}{2}\right),(y, s)\right) .
\end{aligned}
$$

McBryan-Spencer bounds applied to $W_{l}(x, t)$ give:

$$
G_{W_{l}(x, t)}^{(n)}((x, t),(x, s)) \leqq e^{-h(x)|t-s|} .
$$

Therefore

$$
G_{\Omega}^{(n)}((x, t),(y, s)) \leqq G_{\Omega}^{(n)}(\bar{Z},(y, s))\left[2 d \frac{J}{n} \sum_{u \in I_{l}^{(n)}(t)} e^{-h(x)|t-u|}+2 e^{-\frac{h(x) l}{2}}\right],
$$

where $\bar{Z}$ is defined by

$$
\begin{aligned}
G^{(n)}(\bar{Z},(y, s)) & =\max \left\{G_{\Omega}^{(n)}(Z,(y, s)) ; \quad Z=(x, t \pm l / 2)\right. \\
\text { or } \quad Z & \left.=\left(x^{\prime}, u\right), \quad\left|x-x^{\prime}\right|=1, \quad u \in I_{l}^{(n)}(t)\right\}
\end{aligned}
$$

Suppose now $h(x) \geqq \frac{J}{\alpha}>0$ for all $x \in \Lambda$. Then

$$
\begin{aligned}
2 d \frac{J}{n} \sum_{u \in I_{l}^{(n)}(t)} e^{-h(x)|t-u|}+2 e^{-\frac{h(x) l}{2}} & \leqq\left(\frac{4 d J}{h(x)}+2 e^{-\frac{h(x) l}{2}}\right) \\
& \leqq 4 d \alpha+2 e^{-\frac{J l}{\alpha}}<1
\end{aligned}
$$

provided $4 d \alpha<1$ and $l=l(\alpha)$ is sufficiently large. In this case, with

$$
\begin{aligned}
e^{-m} & =\left(4 d \alpha+2 e^{-\frac{J l}{\alpha}}\right), \\
G_{\Omega}^{(n)}(X, Y) & \leqq e^{-m} G_{\Omega}^{(n)}(\bar{Z}, Y) .
\end{aligned}
$$

Iterating (5.8) we get

$$
G_{\Omega}^{(n)}((x, t),(y, s)) \leqq e^{-m l_{i}(x-y),(t-s) / / \|_{\infty}} .
$$




\section{Multiscale Analysis}

For $x \in \mathbf{Z}^{d}$ and $L>0$ let

$$
\Lambda_{L}(x)=\left\{y \in \mathbf{Z}^{d} ;|y-x| \leqq L\right\},
$$

and for $X=(x, t) \in \mathbf{Z}^{d} \times \frac{\mathbf{Z}}{n}, T>0$,

$$
B_{L, T}^{(n)}(X)=\Lambda_{L}(x) \times\left(\left[t-\frac{T}{2}, t+\frac{T}{2}\right] \cap \frac{\mathbf{Z}}{n}\right) .
$$

With a choice of $q>1$ to be later specified we set

$$
B_{L}^{(n)}=B_{L, L^{q}}^{(n)}(X) .
$$

Definition. A point $x \in \mathbf{Z}^{d}$ is $m$-regular at scale $L$ ( $x$ is $(m, L)$-regular) if, for all $n=1,2, \ldots$,

$$
G_{B_{L}((x, 0))}^{(n)}((x, 0), Y) \leqq e^{-m L}, \quad \forall Y \in \hat{o} B_{L}^{(n)}((x, 0)) .
$$

Remarks. 1) By translation invariance in the time direction if $x$ is $(m, L)$-regular then

$$
G_{B_{L}}^{(n)}((x, t), Y) \leqq e^{-m L}, \quad \forall Y \in \partial B_{L}((x, t)), \quad \forall t \in \frac{\mathbf{Z}}{n}
$$

2) If we define, for $W \subset \mathbf{Z}^{d} \times \frac{\mathbf{Z}}{n}$,

$$
G_{W}^{(n)}((x, t), \partial) \equiv \sum_{Y \in \hat{c} W} \frac{1}{n} G_{W}^{(n)}((x, t), Y),
$$

it follows that if $x \in \mathbf{Z}^{d}$ is $(m, L)$-regular then

$$
G_{B_{L}(x, t)}^{(n)}((x, t), \partial) \leqq e^{-m^{\prime} L}
$$

with

$$
m^{\prime} \geqq m-\frac{c \log L}{L}
$$

where the constant $c$ depends on $q$.

Theorem 6.1. Let $p>2 d$ and suppose that

$\left(\mathrm{H}_{0}\right)$ : There exists $m_{0}>0, L_{0}>0$ such that $\mathbf{P}\left\{0\right.$ is $\left(m_{0}, L_{0}\right)$-regular $\} \geqq 1-1 / L_{0}^{p}$.

Let $L_{k+1}=L_{k}^{\alpha}, k=0,1,2, \ldots$ with $1<\alpha<2 p /(p+2 d)$. Then for any $0<m_{\infty}<m$ there exists $\bar{L}=\bar{L}\left(p, d, q, m_{0}, \alpha, m_{\infty}\right)$ such that if $L_{0}>\bar{L}$ we have

$$
\mathbf{P}\left\{0 \text { is }\left(m_{\infty}, L_{k}\right) \text {-regular }\right\} \geqq 1-\frac{1}{L_{k}^{p}}
$$

for all $k=1,2, \ldots$.

Remark. Assumption $H_{0}$ can be satisfied for any choice of $\left(L_{0}, m_{0}\right)$ by taking $J$ sufficiently small. This follows from the mean-field bounds of Sect. 5 . 
Corollary 6.2. With the hypothesis of Theorem 6.1, given $0<m<m_{\infty}$, there exists a constant $C_{\mathrm{r}, \mathrm{h}, J}<\infty$ such that

$$
G^{(n)}((x, t),(y, s)) \leqq C_{x, \mathbf{h}, J} e^{-m !\left(x-y,|t-s|^{1 / q} \|_{\infty}\right.} .
$$

Proof of Corollary. It is the same as the proof of Corollary 3.2 in [12].

Proof of Theorem 6.1. Let $p>d, 1<\alpha<2 p /(p+2 \alpha)$ and for some $l>0$ let

$$
\mathbf{P}\{0 \text { is }(m, l) \text {-regular }\} \geqq 1-\frac{1}{l^{p}} \text {. }
$$

Then for $L=l^{\alpha}$ we have

$$
\begin{gathered}
\mathbf{P}\left\{\exists x_{1}, x_{2} \in \Lambda_{L}(0), x_{1} \text { and } x_{2}(m, l) \text {-singular with } \Lambda_{l}\left(x_{1}\right) \cap \Lambda_{l}\left(x_{2}\right)=\phi\right\} \\
\leqq \frac{L^{2 d}}{l^{2 p}}=\frac{1}{L^{\frac{2 p}{\alpha}-2 d}}<\frac{1}{2 L^{p}}
\end{gathered}
$$

for $l$ sufficiently large.

It is therefore sufficient to consider the situation where there exists at most one box of side $2 l$ around some point $\mathbf{Z} \in \Lambda_{L}(0)$ such that $X$ is $(m, l)$ regular for all $x \in \Lambda_{L}(0) \backslash \Lambda_{2 l}(Z)$. Let us first estimate $G_{B_{L}(0)}^{(n)}(0, X)$ for $X \in \partial_{V} B_{L}(0)$. From $S L R$ inequalities we get

$$
G_{B_{L}(0)}(0, X)=\prod_{i=0}^{n} G_{B_{l}\left(Z_{l}\right)}\left(Z_{i}, \partial\right) G_{B_{L}(0)}\left(Z_{n}, Z_{m}^{\prime}\right) \prod_{j=0}^{m} G_{B_{l}\left(Z_{j}^{\prime}\right)}\left(Z_{j}^{\prime}, \partial\right)
$$

for some $Z, \ldots, Z_{n}, Z_{1}^{\prime}, \ldots, Z_{m}^{\prime}$, where

$$
Z_{0}=(0,0), Z_{0}^{\prime}=X ; \quad Z_{1}, Z_{2}, \ldots, Z_{n-1}, Z_{1}^{\prime}, \ldots, Z_{m-1}^{\prime} \in B_{L}(0,0) \backslash B_{2 l, L^{q}}((z, 0))
$$

and $Z_{n}, Z_{m}^{\prime} \in B_{L}(0,0)$, with $n+m \geqq \frac{\frac{L}{2}-2 l}{l / 2}=\frac{L}{l}-4$. Therefore,

$$
G_{B_{L}(0)}((0,0), X) \leqq\left(e^{-m^{\prime} l}\right)^{\frac{L}{l}-4} \leqq e^{-M L},
$$

where $M=m^{\prime}-o(l)=m-o(l)$, for large $l$. If now $X \in \partial_{H} B_{L}(0,0)$ we now use the McBryan-Spencer bound (4.5), to get

$$
\mathbf{P}\left\{G_{B_{L}(0,0)}((0,0),(x, t)) \geqq e^{-\frac{|t|}{L^{\tau+d}}}\right\} \leqq \mathbf{P}\left\{h_{L}<\frac{1}{L^{\tau}}\right\}=\mathbf{P}\left\{\frac{1}{h_{L}}>L^{\tau}\right\},
$$

where $h_{L}=h_{\min }^{\left(B_{L}(0,0)\right)}=\min \left\{h(x), x \in B_{L}(0,0)\right\}$. But

$$
\mathbf{P}\left\{\frac{1}{h_{L}}>L^{\tau}\right\} \leqq L^{d} \mathbf{P}\left\{\frac{1}{h(0)}>L^{\tau}\right\} \leqq \frac{L^{d} \mathbf{E}\left(\frac{1}{h(0)^{\delta}}\right)}{L^{\tau \delta}}=\frac{1}{\bar{h}^{\delta}} \frac{1}{L^{\tau \delta-\alpha}}<\frac{1}{2 L^{p}}
$$

if $\tau \delta>p+d$, and $L$ sufficiently large. Therefore, if $X \in \partial_{H} B_{L}(0,0)$, i.e., $X=(x, t)$ with $|t|=\frac{L^{q}}{2}$ we have from (6.11) and (6.12)

$$
\mathbf{P}\left\{G_{B_{L}(0,0)}((0,0), X) \leqq e^{-\frac{L^{q}}{2 L^{\tau+\alpha}}}\right\} \geqq 1-\frac{1}{2 L^{p}}
$$


If we now take $q>\tau+d+1$, and $L$ sufficiently large,

$$
\mathbf{P}\left\{G_{B_{L}(0,0)}((0,0), X) \leqq e^{-M L}\right\} \geqq 1-\frac{1}{2 L^{p}}
$$

if $X \in \partial_{H} B_{L}(0,0)$. Putting together (6.14), (6.10) and (6.8) we find that

$$
\mathbf{P}\{0 \text { is }(M, l) \text {-regular }\} \geqq 1-\frac{1}{L^{p}},
$$

which concludes the proof.

\section{References}

1. a) Abeles, B.: Phys. Rev. B 15, 2828 (1977)

b) Doniach, S.: Phys. Rev. B 24, 5063 (1981)

c) Ma, M., Halperin, B.I., Lee, P.A.: Phys. Rev. B 34, 3136-3146 (1986)

d) Jacobs, L., Jose, J.V.: Physica B 152, 148-155 (1988)

e) Fisher, M.P.A., Grinstein, G.: Phys. Rev. Lett. 60, 208 (1988)

f) Fisher, M.P.A., Weichman, P.B., Grinstein, G., Fisher, D.S.: Phys. Rev. B 40, 546-570 (1989)

g) Kim, S., Choi, M.Y.: Phys. Rev. B 41, 111 (1990)

2. Klein, A., Perez, J.F.: Localization in the Ground State of the One-Dimensional $X-Y$ Model with a Random Transverse Field. Commun. Math. Phys. 128, 99-108 (1990)

3. Campanino, M., Klein, A., Perez, J.F.: Localization in the Ground State of the Ising Model with a Random Transverse Field. Commun. Math. Phys. 135, 499-515 (1991)

4. von Dreifus, H.: Ph.D. Thesis, New York University, 1987

5. von Dreifus, H., Klein, A.: Commun. Math. Phys. 124, 283-299 (1989)

6. Driessler, W., Landau, L.J., Perez, J.F.: Estimates of Critical Lengths and Critical Temperatures for Classical and Quantum Lattice Systems. J. Stat. Phys. 20, 123-162 (1979)

7. McBryan, O.A., Spencer, T.: Commun. Math. Phys. 53, 299 (1976)

8. Bellissard, J., de Angelis, G.F.: Gaussian Limit of Compact Spin Systems, in: Random Fields. Fritz, J., Lebowitz, J., Szás (eds.), Vol. I, Amsterdam: North Holland 1979

9. Ginibre, J.: Commun. Math. Phys. 16, 310 (1970)

10. a) Simon. B.: Correlation Inequalities and the Decay of Correlations in Ferromagnets. Commun. Math. Phys. 77, 127 (1980)

b) Lieb, E.H.: A Refinement of Simon's Inequality. Commun. Math. Phys. 77, 11 (1980)

c) Rivasseau, V.: Lieb's Correlation Inequalities for Plane Rotors. Commun. Math. Phys. 77, 145-147 (1980)

11. Reed, M., Simon, B.: Methods of Modern Mathematical Physics, Vol. IV. New York: Academic Press 1970

12. Campanino, M., Klein, A.: Decay of Two-Point Functions for $(d+1)$-Dimensional Percolation, Ising and Potts Models with $d$-Dimensional Disorder. Commun. Math. Phys. 135, 483-497 (1991)

13. Aizenman, M., Klein, A., Newman, C.: (in preparation)

14. Bonato, C.A., Perez, J.F.: J. Stat. Phys. 56, 13-22 (1989)

15. Aizenman, M., Simon, B.: Commun. Math. Phys. 77, 137-143 (1980)

16. Reed, M., Simon, B.: Methods of Modern Mathematical Physics, vol. III. New York: Academic Press 1970

17. Klein, A.: Extinction of Contact and Percolation Processes in a Random Environment. Preprint 